\title{
大基数性质与险峻理想
}

\author{
张 再 跃 \\ (中国科学院软件研究所, 北京 100080)
}

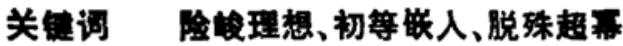

\section{1 基本概念和术语}

本文采用集论的标准概念和术语. $\kappa$ 表示正则不可数基数, $\lambda$ 为 $\geqslant \pi$ 的基数, $I$ 是 $P_{k x}$ 上的理想则意味着 $I$ 是 $\kappa$-完全、非主并且好的理想. 如果 $\mathscr{M}$ 是 $Z F C$ 的基 模 型, 那么 $U l t_{\mathscr{U}}(\mathscr{M})$ 表示相时 $\mathscr{U}$ 的 $\mathscr{M}$ 超幕, 其中 $\mathscr{U}$ 是 $P_{k} \lambda$ 上的超滤.

设 $I$ 是 $P_{k} \lambda$ 上的理想, 我们考虑用 Boolean 代数 $P\left(P_{k} \lambda\right) / I$ 的完备建立的 $\mathscr{M}$ 的脱殊 扩充, 即: 用 $\left\langle R(I), \leqslant_{I}>\right.$ 为力迫条件, 其中 $R(I)=\left\{x \subseteq P_{\alpha} \lambda: x \in I\right\}, x \leqslant{ }_{1} y$ iff $x \subseteq y$, 令 $W_{l}$ 是 $R(I)$ 上的脱殊滤子, 则 $W_{I}$ 是 $P_{\kappa} \lambda$ 上 $\mathscr{M}-k$-完全的 $\mathscr{M}$-超滤. 根据超幂基本定理 知 $U l_{t_{W}}(\mathscr{M})$ 是 $Z F C$ 的模型. 令 $i: \mathscr{M} \rightarrow U l_{t_{I}}(\mathscr{C})$ 为自然初等埌入, 即 $j(x)=\left[C_{x}\right]$, 其中 $C_{x}$ 为 $P_{x} \lambda$ 上取常值 $x$ 的函数. 当 $U l_{W_{1}}(\mathscr{M})$ 良基, 我们用它的传递降幂标识它.

定义 $P_{\star} \lambda$ 上的理想 $I$ 称为险峻的, 如果对 $R(I)$ 上任意的脱殊 $W_{I}, U l_{t_{W_{l}}}(\mathscr{M})$ 良 基 ${ }^{[1]}$.

在本文中, 常用力迫概念, 设 $P$ 为偏序集, $G$ 是 $P$ 上的脱殊集, 则 $\mathscr{M}[G]$ 表示基模的脱 殊扩充.当 $x \in \mathscr{M}[G]$ 时,我们用黑体 $\boldsymbol{x}$ 表示其 Boolean 值名,如果 $x \in \mathscr{M}$,它的标准名用 $\check{x}$ 表示, $i_{G}$ 定义为 $\mathscr{M}^{B}$ 的 $G$-解释函数, 其中 $B=r . o(P)$. 显然当 $x \in \mathscr{M}$ 时 $i_{G}(\check{x})-x$. 我们将用到的一个基本事实是，基模良基的充要条件是它的脱殊扩充良基.

关于更多这方面的知识可参阅文献 [2].

\section{2 基本性质和引理}

Solovay ${ }^{[3]}$ 中考虑了由理想生成理想的性质, 下面定理对超滤生成理想性质进行了刻划.

定理 1 设 $\mathscr{U}_{\lambda}$ 是 $P_{\star} \lambda$ 上 $\kappa$-完全好的超滤, $P$ 是满足 $\kappa$-chain condition $(\kappa-c, c)$ 的偏序, $G$ 是 $P$ 上的脱殊集,则在 $\mathscr{M}[G]$ 中由 $\mathscr{U}_{\lambda}$ 生成的理想是 $P_{\star} \lambda$ 上 $\kappa$-完全, 非主并 且好的理想.

证 在 $\mathscr{K}[G]$ 中 $\mathscr{U}_{2}$ 生成的理想 $l$ 定义如下:

$$
x \in I \text { iff } \exists y \in \mathscr{U}_{2}, x \cap y=\varnothing,
$$

不难验证, 如此定义的 $I$ 是 $P_{k} \lambda$ 上的理想,关于 $\kappa$-完全性证明如下: 令 $\mathscr{R}=\left\{x_{\xi}: \xi<r\right\}$ 是 $I$ 的少于 $\kappa$ 个元素的族, 令 $p_{0} \in G, p_{0} I+\forall \xi<\check{r} x_{\xi} \in I$. 根据 $I$ 的定义, 对每个 $\xi<r$ 以及每个 $p \leqslant p_{0}$, 存在 $q \leqslant p$ 以及某个 $y \in \mathscr{U}_{2}, q H \check{x}_{\xi} \cap \check{y}-\varnothing$. 令 $W_{\xi}$ 是满足下列条件的极大不可 
此族: $q \leqslant p_{0}$, 有 $y_{\xi, q} \in \mathscr{U}{ }_{2}$ 满足 $q 1+\check{x}_{\xi} \cap \check{y}_{\xi, q}-\phi$. 由于 $P$ 是 $\kappa-c . c$ 的, 故 $\left|W_{\xi}\right|<\kappa$, 因 此 $y-\cap\left\{y_{\xi, q}: \xi<r\right.$ 并且 $\left.q \in W_{\xi}\right\}$ 属于 $\mathscr{U} \alpha_{\lambda}$. 容易检证 $p_{0} H+\left(\underset{\xi<r}{U \check{x}_{\xi}}\right) \cap \check{y}-\varnothing$. 根据 $I$ 的 定义知 $U \mathscr{X} \in I$. 关于 $I$ 的其它性质可由 $\mathscr{U}_{\lambda}$ 的相应性质直接得到. 证毕.

令 $\kappa$ 是 $\mathscr{M}$ 中不可及基数, $\lambda \geqslant \kappa, \mathscr{U}{ }_{\lambda}$ 是 $P_{\alpha} \lambda$ 上的超滤. 在 $\mathscr{M}$ 中定义 $P$ 如下:

对每个 $p \in P, p$ 是函数, 满足

$$
\begin{aligned}
& \operatorname{dom}(p) \subseteq \omega \times \kappa \text { 并且 }|\operatorname{dom}(p)|<\omega, \\
& p(n, \alpha)<\alpha(\text { 对任意 }(n, \alpha) \in \operatorname{dom}(p)),
\end{aligned}
$$

$p, q \in P$, 记 $q \subset p$ 为 $p<q$.

根据文献 $[2] 引$ 引理 $20.4,(P,<)$ 满足 $\kappa-c . c$ 并且如果 $G$ 是 $P$-脱殊集, 则在 $\mathscr{M}[G]$ 中 $\hbar=\aleph_{1}$. 在 $\mathscr{M}[G]$ 中考虑, 由于 $\mathscr{U}_{\lambda}$ 是 $P_{x} \lambda$ 上的超滤, 则有初等㰩人 $j: \mathscr{M} \rightarrow \mathscr{N}(\approx$ $\left.U l_{t_{\alpha} \lambda}(\mathscr{C})\right) . \kappa$ 是最小改变数 ${ }^{[4]}$. 因此对 $p \in P, j(p)-p$. 如果 ${ }^{*} G^{*}$ 是任意 $j(P)$-脱殊集 满足 $G^{*} \supseteq G$, 则我们有

引理 1 初等 $j: \mathscr{M} \rightarrow \mathscr{N}$ 可被扩充为 $\mathscr{M}[G] \rightarrow \mathscr{N}\left[G^{*}\right]$ 的初等嵌人 $j^{*}$.

证 对任意 $x \in \mathscr{M}[G]$ 定义 $j^{*}(x)=i_{G^{*}}^{*}(j(x))$. 因为 $x \in \mathscr{M}^{B}(B=r .0(P))$, 则 $j(\boldsymbol{x}) \in \mathscr{N}^{B}$ 这样 $i_{G}^{*}(j(\boldsymbol{x})) \in \mathscr{N}\left[G^{*}\right]$. 令 $y$ 为 $\mathscr{M}[G]$ 中任意元, $p \in G, p H \boldsymbol{x}=\boldsymbol{y}$ 在 $\mathscr{M}$ 中 成立, 利用 $j$ 的初等性质, 在 $\mathscr{N}$ 中有 $j(p)+j(\boldsymbol{x})=j(\boldsymbol{y})$, 而 $j(p)=p \in G \subseteq G^{*}$ 所以在 $\mathscr{N}$ 中 $p+\underset{j(P)}{j(x)}=j(\boldsymbol{y})$, 即 $i_{G}^{*}(j(\boldsymbol{x}))=i_{G}^{*}(j(\boldsymbol{y}))$ 由此证明了 $j^{*}$ 是确切定义的. 现设 $\varphi$ 是任一公式 满足 $\mathscr{M}[G] \models \varphi(x, \cdots)$, 令 $p \in G p H+\varphi(x \cdots)$, 在 $\mathscr{N}$ 中 $\underset{j(p)}{p} \varphi(j(x) \cdots)$. 因此 $\mathscr{N}\left[G^{*}\right] H$ $\varphi\left(j^{*}(x), \cdots\right), j^{*}$ 是初等. 证毕.

\section{3 主要结果及证明}

定理 2 如果 $\kappa$ 强紧与 $Z F C$ 协调, 则对任意 $\lambda \geqslant \kappa$ 有 $Z F C$ 的模型, 在其中 $\kappa=\aleph_{1}$, 并且 $P_{k} \lambda$ 载有险峻理想.

证令 $\mathscr{A}$ 是基模. $\boldsymbol{\kappa}$ 是强紧基数, $\lambda \geqslant \kappa, \mathscr{U}_{\lambda}$ 是 $\mathscr{M}$ 上 $P_{\kappa} \lambda$ 上的超滤, 用(2.1)定义 的 $P$ 为力迫条件, $G$ 是 $P$-脱殊集, 则在 $\mathscr{M}[G]$ 中 $\kappa=\aleph_{1}$, 用 $\mathscr{N}$ 表示 $U l_{\boldsymbol{C l}_{\alpha}}(\mathscr{M}), j: \mathscr{M} \rightarrow$ $\mathscr{N}$ 是自然初等嵌人, 在 $\mathscr{M}[G]$ 设 $l$ 是 $\mathscr{U}$ 入 生成的理想,即

$$
x \in I \text { iff } \exists y \in \mathscr{U}_{2}, x \cap y=\varnothing \text {. }
$$

根据定理 $1, I$ 在 $\mathscr{M}[G]$ 中是可数完全的, 我们证明 $I$ 是险峻的, 为此我们只要 证明在 $\mathscr{M}[G]$ 中对每个 $x \in P\left(P \_\lambda\right) / I$, 有 $R(I)$-脱殊超滤 $W$, 使得 $x \in W$ 并且 $U l t_{W}(\mathscr{M}[G])$ 是良基的.

根据引理 1 , 如果 $G^{*}$ 是任何 $j(P)$-脱殊集, 并且 $G \subseteq G^{*}$, 则有 $j$ 的自然扩充

$$
j^{*}: \mathscr{M}[G] \rightarrow \mathscr{N}\left[G^{*}\right] j^{*}(x)=i_{G}^{*}(j(x))(x \in \mathscr{M}[G]),
$$

并且 $j^{*}$ 是初等的. 令 $H$ 是 $\mathscr{N}$ 中由 $P_{k} \lambda$ 上的恒等函数 $d(s)=s\left(s \in P_{k} \lambda\right)$ 表示的元素, 则 $x \in \mathscr{U}_{\lambda}$ iff $H \in j(x)$. 对任意 $y \in \mathscr{M}[G]$, 我们断言: $y \in I$ iff $\exists p \in G,\left\{s: p H \leftarrow\llcorner\in y\} \in \mathscr{U} \lambda_{\lambda}\right.$.

断言证明: $y \in I$ iff $\exists p_{0} \in G$ 并有某个 $z \in \mathscr{U}_{2}$ 使得 $p_{0} H+\boldsymbol{y} \cap \check{z}=\varnothing$, 则必有某个 $p \leqslant$ $p_{0}(p \in G) p H+y \in \check{u}_{\lambda}$. 否则对任意 $q \leqslant p_{0}(q \in G)$ 不能力迫 $y \in \mathscr{U}_{\lambda}$, 于是必有某个 $q \leqslant$ $p_{0}(q \in G) q+y \in \check{u}_{\lambda}$ 则 $q H \boldsymbol{y} \cap \check{z} \neq \varnothing$ 和 $y \cap z=\varnothing$ 矛盾. 现设 $p \leqslant p_{0}(p \in G) p H+\boldsymbol{y} \check{u}_{\Lambda}$, 则有 $p H+\check{H} \in j(y)$ i.e $\{s: p H \check{s} \in y\} \in \mathscr{U}{ }_{2}$. 反之, 如果 $y \in I$ 则 $y \cap\{s: p H \check{s} \in \boldsymbol{y}\} \neq \varnothing$ 矛活， 
故断言成立.

根据断言, 我们有 $y \in I$ 等价于 $\exists p \in G p \underset{i(P)}{ } \rightarrow \in j^{*}(y)$. 如 果 $x_{0} \in I$ 则有 $q_{0} \in j(P), q_{i} \mid+$ $H \in j^{*}\left(x_{0}\right)$. 令 $G^{*} \supseteq G \cup\left\{q_{0}\right\}$ 是 $j(P)$-脱殊集. 定义 $P_{x} \lambda$ 上的 $\mathscr{M}[G]$-超滤 $W$ 如下: $x \in W$ iff $H \in i_{G}^{*}(i(x))$,

因为 $p \in G, j(p)=p \in G^{*}$, 上式不依赖于 $\boldsymbol{x}$ 名的选取, 故 $W$ 的定义是确切的, 而 $q_{0} \in G^{*}$, 故 $x_{0} \in W$.

要完成定理的证明,我们将证明以下两个引理:

引理 $2 U l_{W}(\mathscr{A}[G])$ 是良基的。

证 令 $h: U l_{W}(\mathscr{M}[G]) \rightarrow \mathscr{N}\left[G^{*}\right]$ 如下定义: $h\left([f]_{W}\right)=j^{*}(f)(H)\left(\right.$ 对任意 $\left.[f]_{W} \in U l_{W}(\mathscr{M}[G])\right)$, 我们直接验证 $h$ 是同构的,再根据 $\mathscr{N}\left[G^{*}\right]$ 的良基性质可得 $U l_{W}(\mathscr{M}[G]$ ) 是良基的.

引理 $3 W$ 是 $\mathscr{A}[G]-$ 脱殊集.

证 只要证明对任意 $I$-分割 $\mathscr{X}=\left\{x_{i} i<\theta\right\} \mathscr{R} \cap W \neq \varnothing$. 反证, 假设 对每个

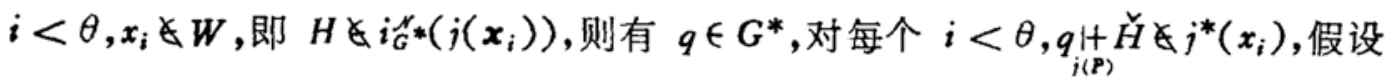

$$
\left[<q_{s}: s \in p_{\kappa} \lambda>\right]_{\alpha_{\alpha}}=q
$$

并且 $T=\left\{s: g_{s} \in G\right\}$. 如果我们证明 $T \in I, T \cap x_{i} \in I$ (对 $\forall i<\theta$ ) 则产生和 $\mathscr{X}$ 的极大性的

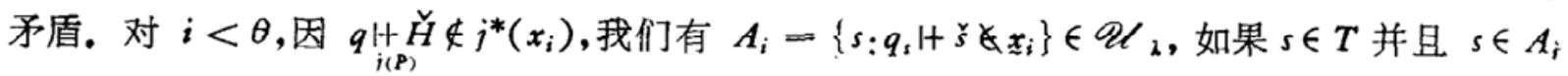
则 $q_{s} \in G, q_{s} 1+s \notin x_{i}$, 由此便有 $s \notin x_{i}$, 即 $\left(T \cap x_{i}\right) \cap A_{i}=\varnothing$, 根据 $I$ 的定义知 $T \cap x_{i} \in I$. 要 证 $T \nless I$, 我们只要证对任意 $y \in \mathscr{U}_{\lambda}, T \cap y \neq \varnothing$. 固定 $y \in \mathscr{U}_{\lambda}$, 令 $A=\left\{q_{s}: s \in y\right\}$ 不失一 般性, 我们可假定 $A$ 包含 $P$ 的分割, 这是因为 $P$ 满足 $\kappa-c, c$ 而 $y$ 在 $P$ 。 $\lambda$ 上无界, 改变小于 $\kappa$ 个 $q$, 不影响 $q$ 的性质, 现令 $D=\{p \in P: p \leqslant q$, 对某个 $s \in y\}$, 我们证 $D$ 在 $P$ 中稠密. 任取 $p_{0} \in P$, 则 $p_{0}$ 与某个 $q_{s}$ 可比, 从而有 $p \leqslant p_{0}, q_{s}$ 故 $p \in D$. 于是 $G \cap D \neq \varnothing$. 令 $p \in G \cap D$ 则 $p \in G$ 并且对某个 $s \in y, p \leqslant q_{s}$. 因此 $q_{s} \in G, s \in T i, e T \cap y \neq \varnothing$. 证毕.

定理 $3^{[\lambda]}$ 如果 $P_{s} \lambda$ 载有险峻理想, 则对任意 $\lambda$ 的有界子集 $b, b^{\#}$ 存在

证设 $I$ 是 $P_{\kappa} \lambda$ 上的险峻理想, $b \subset \lambda$ 为有界子集, 令 $\delta=\max \left\{(\sup (b))^{+}, \kappa^{+}\right\}, W$ 是 $P\left(P_{\kappa} \lambda\right) / I$-脱殊集, 则 $U l_{W}(\mathscr{M})$ 良基. 令 $j: \mathscr{M} \rightarrow \mathscr{N}\left(\approx U l_{t_{W}}(\mathscr{M})\right.$ 为自然初等唄人,考 虑函数 $f: P_{\alpha} \lambda \rightarrow \mathscr{C}$ 满足 $|\mathrm{range}(f)|<\delta$, 这此函数的等价类形成的超幂用 $U l t_{\mathrm{W}}^{*}(\mathscr{M})$ 表 示, 它显然是良基的. $\mathscr{N}^{*}$ 表其传递降幂, $[f]^{*}$ 表示 $f$ 刻划的元素, $i: \mathscr{M} \rightarrow \mathscr{N}^{*}$ 为标准虾 人,则 $i$ 是初等的并且下述事实成立:

1) 对任意 $f: P_{\kappa} \lambda \rightarrow \delta$, $\mid$ range $(f) \mid<\delta$, 有 $[f]-[f]^{*}$;

2) 如果 $x$ 是 $\delta$ 的有界子界, 则 $i(x)-j(x)$, 特别 $i(b)-j(b), i(\alpha)=j(\alpha)(\forall \alpha<\delta)$;

3) $i(\delta)<j(\delta)$.

定义 $h: \mathscr{N}^{*} \rightarrow \mathscr{N}, h\left([f]^{*}\right)=[f]$, 则 $h$ 为初等嵌人,并有下图可交换:

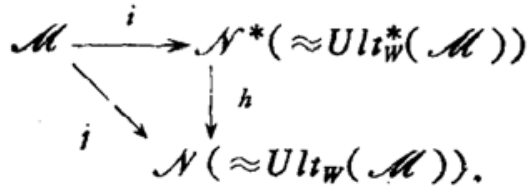

由 3) 知 $h$ 改变 $i(\delta), 2)$ 以及上述图的交换性给出 $h(i(b))=j(b)-i(b)$, 国此 
$h$ 是一个非平凡初等嵌人 $(L[i(b)] \rightarrow L[i(b)])$, 因此 $i(b)$ \#存在, 从而 $U l_{t_{W}}(\mathscr{L})$ $\vDash j(b) \#$ 存在. 利用初等性 $\mathscr{A} \vDash b^{\#}$ 存在. 证毕.

推论 如果 $\kappa$ 是 $\lambda$-强紧的, 则有 $Z F C$ 的模型在其中, 对任意 $\lambda$ 的有界子界 $b, b^{*}$ 存在.

证由定理 2 和定理 3 直接可得.

\section{参文文献}

[1] Matisubara, Y., Ann. of Pure and Applied Logic, 1987, 36: 225-234.

[2] Tech, T., Set Theory, Academic Press, 1978.

[3] Solovay, R., Proceedings of Symposia in Pure Matke., Vol 13, Part I (ed. Scott, D.), Amer. Mathe. Soc., Providence, R. 1., 1971, 397-428.

[4] Solovay. R., Ann. of Mathe. Logic, 1978. 13: 73-116. 